\title{
Relaciones sociales duraderas entre tripulación de cruceros y población residente, análisis del caso Cozumel desde la teoría del intercambio social
}

\author{
Erick David García González* \\ Universidad Intercultural del Estado de Puebla (México)
}

Alfonso González Damián**

Universidad de Quintana Roo (México)

\begin{abstract}
Resumen: Hasta la fecha, pocos son los trabajos en los que se han abordado problemáticas relacionadas con los tripulantes de cruceros. Además, éstas no han sido explicadas desde una teoría que permita una mejor comprensión de los efectos generados por dicho actor en los sitios donde desembarcan durante su tiempo libre. Por lo anterior, el presente trabajo consiste en explicar la relación entre los tripulantes de cruceros y los residentes locales de Cozumel a partir de la teoría del intercambio social, a fin de conocer de mejor forma la interacción entre ellos y poder determinar desde dicha teoría su relevancia en el destino. El análisis realizado muestra que si bien existe una relación costo-beneficio entre ambos actores, también son notorias las relaciones duraderas con intercambios recíprocos que han culminado en diversos efectos interculturales en Cozumel, mismos que merecen ser estudiados con mayor profundidad en trabajos futuros. Lo anterior puede permitir reflexionar a gestores y académicos sobre la importancia del tripulante de crucero como actor en los puertos de cruceros.
\end{abstract}

Palabras Clave: Cruceros; Tripulantes; Cozumel; Teoría del Intercambio Social.

Long-lasting social relationships between cruise ship-crew and local residents, the case study of Cozumel from the perspective of social exchange theory

Abstract: Up to this date, there are few articles that deal with the problems faced by cruise ship crew members. Furthermore, said problems, when dealt with, do not engage with the problems within a theoretical framework that might permit a better comprehension of the effects caused by these agents when they come off the ships on their free time. For those reasons, the present research focuses on explaining the relations between the cruise-ship crew-members and local residents of the island of Cozumel using Social Exchange Theory to analyse their interaction and the relevance of the same to the destination. The findings demonstrate that even when there is a cost-benefit interaction, long-lasting relationships with reciprocal exchanges are notable, leading to diverse intercultural effects in Cozumel that must be studied in depth in future research. The preliminary information should make local managers and researchers reflect on the potential importance of cruise ship crew members as agents in cruise ports.

Keywords: Cruises; Cruise Ship Crew Members; Cozumel; Social Exchange Theory.

\section{Introducción}

Al paso de los años, el turismo de cruceros se ha consolidado como un fenómeno de crecimiento constante a nivel mundial (Wu, 2005; Dowling, 2006; Gibson, 2008; Klein, 2011). Para ejemplificar

Profesor Investigador de Tiempo Completo, Universidad Intercultural del Estado de Puebla, Mexico; E-mail: erick.garcia@ uiep.edu.mx

** Departamento de Estudios Sociales y Empresariales, Universidad de Quintana Roo, Mexico; E-mail: gonzalezd@uqroo.edu.mx 
lo anterior, se puede mencionar que en el año 2015, hubo un total de 23 millones de cruceristas (The Florida-Caribbean Cruise Association FCCA, 2015). De acuerdo con la asociación Internacional de Líneas de Cruceros (CLIA por sus siglas en inglés) en 2017, se contabilizaron un total de 60 diferentes líneas de cruceros a escala global, siendo Carnival Corporation (48.4 \%), Royal Caribbean Cruises Limited (23.3\%) y Norwegian Cruise lines (7.6\%) las tres firmas que figuran como las más sobresalientes en cuestión del dominio de la industria (Petrick y Durko, 2015).

Diversos son los factores que se compaginan para formar el éxito de la industria de cruceros, entre ellos, se encuentra la facilidad que tienen los cruceristas de conocer en poco tiempo distintos destinos turísticos sin la necesidad de utilizar diversos medios de transporte (Dowling, 2006), los lujos ofrecidos abordo, la capacidad de poder disfrutar de alimentos y bebidas de distintas partes del mundo en un mismo lugar, la oferta de shows en vivo y diversas actividades recreativas, así como excursiones a los atractivos de los puertos de escala (Gibson, 2008; Kerswill y Mair, 2015). Sin embargo, es necesario mencionar que lo anterior es posible gracias a las diversas labores y funciones de los trabajadores de los cruceros, mismos que son conocidos como tripulantes o crew members (Gibson, 2008; García, 2015).

La asociación Internacional de Líneas de Cruceros menciona que hasta el año 2017 existen más de 900 mil empleos generados por la industria de cruceros a nivel mundial, de los cuales se estima que alrededor de 223,914 tripulantes trabajando a bordo de los barcos (http://www.crew-center.com) distribuidos en tres tipos de jerarquías. Los altos mandos se conforman por los capitanes, oficiales y puestos gerenciales que generalmente son ocupados por tripulantes provenientes de países de Norteamérica y Europa Occidental (Lois, Wang, Wall y Ruxton, 2004). La jerarquía media, compuesta por asistentes y el staff de entretenimiento y animación, suele provenir de Europa del este, mientras que los trabajadores de puestos operativos suelen ser de países asiáticos y latinoamericanos en vías de desarrollo (Zhao, 2002; Weaver, 2005; Wood, 2000; Véronneau y Roy, 2012). Lo anterior conlleva a que en un mismo crucero existan trabajadores de más de 90 nacionalidades diferentes, siendo en su mayoría, provenientes de Filipinas, Italia, Gran Bretaña, Honduras, India, Indonesia, Alemania y Ucrania (Wu, 2005), resultando en evidencia de que el fenómeno de la globalización se ha extendido hacia los mares del mundo debido a los cruceros (Wood, 2000; Gibson, 2008).

A bordo, las altas jerarquías gozan de mayores privilegios, como pueden ser camarotes individuales o bares exclusivos (Zhao, 2002), así mismo, existe una mayor carga laboral para los puestos operativos, reflejado en las altas jornadas laborales que van de las 10 a 14 horas, siete días a la semana (Wood, 2000; Zhao, 2002; Klein, 2003; Weaver, 2005). Lo anterior ha ocasionado que diversos escritos relacionados con el tripulante de crucero, se centren en discutir sobre la mejora en las condiciones laborales y los derechos con los que cuentan como trabajadores del barco (Zhao, 2002; Klein; 2003; Dowling, 2006; Boy y Neumann, 2012; Polat, 2015).

Otros trabajos en los que se mencionan problemáticas relacionadas con el tripulante de crucero, están focalizados en las diversas maneras en las que un tripulante puede influir en los cruceristas al elegir la visita de los atractivos turísticos de un puerto, concluyendo que el crucerista tiende a confiar más en fuentes oficiales que en la publicidad de boca en boca del tripulante (Lloyd, Henry y Thyne, 2011); las motivaciones del tripulante para desembarcar durante su tiempo libre en los puertos que el barco visita, también han sido abordadas, mostrando que el deseo de ingerir alimentos y bebidas fuera del barco, así como conocer distintos lugares del mundo, fungen como las razones principales para desembarcar (Henry y Thyne 2007; Gibson, 2008).

Si bien se demuestra que el tripulante de crucero ha sido visto como sujeto de estudio, aún existen incógnitas relacionadas con su comportamiento en los puertos que visitan (Klein, 2003; Van Broeck y Dierckx, 2011; García, 2015), y aunque los efectos sociales, económicos y ambientales del turismo de cruceros han sido extensamente estudiados (Johnson, 2002; Gibson y Bentley,2006; Martínez, 2008; Brida y Zapata, 2010; Klein, 2011; Santander y Ramos, 2011; Kerswill y Mair, 2015; Palafox, Aguilar y Anaya, 2015; Polat, 2015), existe poca información acerca de los efectos específicos que genera el tripulante de crucero durante su tiempo libre en los puertos, misma que generalmente es homogeneizada con la de los cruceristas al ser ambos definidos como pasajeros (Thyne, James y Lloyd, 2015; Brida y Zapata, 2010; Klein, 2011).

\section{Cozumel como destino de cruceros}

La isla de Cozumel, pertenece a la entidad federativa de Quintana Roo, México. Es la tercera isla más grande del país y la más poblada al contar con 86,415 habitantes, de los cuales 42,577 son de género masculino y 43,838 del femenino (Instituto Nacional de Estadística y Geografía, 2015). 
A inicios de los años setenta, ya se consideraba a la isla como un destino turístico mexicano importante. La relación de Cozumel con los cruceros, comenzó cuando en 1971 el crucero Ariadne atracó por primera vez para posteriormente hacer visitas recurrentes al destino, esto motivó que el trasatlántico Bolero fijara a este sitio dentro de su itinerario en 1974, incitando así a otros cruceros a considerar a la isla como puerto de escala, generando con el paso del tiempo, la necesidad de la construcción del Muelle Internacional en 1981 (Palafox y Zizumbo, 2009). Para el año 1988, fue necesario edificar un segundo puerto de arribo, el cual recibió el nombre de Puerta Maya. En el año 2002, debido a la constante demanda de cruceros, se erigió un tercer puerto (Punta Langosta), evidencia de la recurrente visita y el potencial de la isla como un importante puerto de cruceros (ídem).

De acuerdo con la información brindada por la consultora Business Research \& Economic Advisors (BREA, 2015), Cozumel es el segundo destino de cruceros más visitado del Caribe al recibir un total de 2, 538,100 visitantes (ver tabla 1.1). Así mismo, la Administración Portuaria Integral de Quintana Roo (APIQROO, 2015) argumenta que hubo un total de 1079 cruceros que atracaron en la isla en el año 2015, mostrando así la popularidad de dicho puerto de escala entre los cruceristas.

Tabla 1.1: Los 5 destinos más visitados por el turismo de cruceros en el caribe.

\begin{tabular}{|c|c|}
\hline País/Región & Número Total de Visitas \\
\hline 1. - Bahamas & $2,940,000$ \\
\hline 2. - Cozumel & $2,538,100$ \\
\hline 3. - San Martín & $1,854,400$ \\
\hline 4. - Islas vírgenes & $1,839,700$ \\
\hline 5. - Islas Caimán & $1,446,300$ \\
\hline
\end{tabular}

Fuente: Elaboración propia, basada en BREA, 2015.

Sin embargo, es necesario mencionar que Cozumel también es un destino popular entre los tripulantes y que la cifra de visitas ha ido en aumento, ya que la consultora BREA, contabilizó en 2007 un total de 432,400 trabajadores de crucero visitantes, mientras que para el año 2015, la cifra aumentó a 533.381, siendo hasta ese mismo año, el segundo destino más visitado por tripulantes en la región del Caribe (ver tabla 1.2).

Tabla 1.2: Los 5 destinos en los que desciende mayor cantidad de tripulantes

\begin{tabular}{|l|c|}
\hline \multicolumn{1}{|c|}{ País/Región } & Total de Visitas de Tripulantes (Miles) \\
\hline 1. - Bahamas & 944.9 \\
\hline 2. - Cozumel & $\mathbf{5 3 3 . 4}$ \\
\hline 3. - San Martín & 377.4 \\
\hline 4. - Islas Vírgenes & 351.1 \\
\hline 5. - Jamaica & 244.3 \\
\hline
\end{tabular}

Fuente: Elaboración propia, basada en BREA, 2015.

Así mismo, el trabajo de Van Broeck y Dierckx (2011) describe que debido a que los cruceros mantienen una ruta en la que los barcos atracan en los mismos destinos, la visita de los tripulantes es una constante en la isla de Cozumel. Lo anterior, resulta en diversas actividades entre las que destacan comer, realizar compras, comunicarse con seres queridos a través de internet, visita a los atractivos, consultas médicas y dentales, conseguir suvenires e incluso tener relaciones sexuales (Van Broeck y Dierckx, 2011; García, 2015). Si bien esto puede derivar en un continuo contacto con residentes locales, es necesario profundizar en cómo es que éste inició y cuáles han sido los efectos que han surgido a partir de dicha interacción. 


\section{Teoría del intercambio social y turismo}

Los impactos en la sociedad, resultado de la presencia de actividad turística, han sido analizados desde diversos enfoques teóricos, uno de los más utilizados ha sido el de la teoría del intercambio social, la cual plantea que toda conducta humana social, es decir, toda relación entre individuos, es en última instancia un intercambio, el cual se presenta en tanto ambas partes esperan obtener recompensas de la relación y se mantiene si sus esperanzas se confirman (Hommans, 1961). El concepto central del intercambio de recompensas o valores como base de la interacción, en la literatura teórica se puede indentificar bajo supuestos generales del hedonismo y del individualismo (Blau, 1964; Edwards, 1954; Homans, 1961; Thibaut y Kelly, 1959), concentrada en la operación de variables económico-utilitarias como la maximización individual de las ganancias (Axelrod, 1984; Dugatkin, 1977) o en el planteamiento de una dimensión social de la conducta social desde perspectivas ya no individuales sino interconductuales (Kantor, 1959; Ribes y López, 1985; Ribes 2001).

Un elemento central que permite comprender las interacciones entre individuos es el concepto de reciprocidad, que se presenta como un continuo entre reciprocidad neutra o indiferencia y reciprocidad positiva (Ribes y Pulido, 2015), esta última hace referencia a interacciones en las que los individuos participan puesto que esperan recibir una recompensa, positiva, o evitar un castigo, a cambio de su participación. Tales recompensas o castigos se entienden en un sentido social, es decir, de pertenencia y adhesión al grupo social o al medio en el cual se presenta la relación. El intercambio como tal puede presentarse de manera objetiva con el intercambio directo de bienes y/o acciones, o de manera indirecta, en el que las recompensas o castigos son otorgados o sancionados por la estructura social en su conjunto o por actores - agentes - con mayor poder en la estructura social. En este sentido, en el intercambio social no solo se restringe el intercambio directo que constituye una compra-venta de bienes o servicios, sino al intercambio de valor social en el que el lenguaje se posiciona como vínculo y fondo de las prácticas institucionalizadas (Ribes y Pulido, 2015).

En el ámbito del turismo, la teoría del intercambio social se ha utilizado para explicar los efectos de la actividad en las comunidades receptoras de turismo, desde el trabajo seminal de Ap (1992) y se ha desarrollado de manera sostenida y consistente (Monterrubio, Sosa y Josiam, 2014). Cuando se trata de turismo, se parte de la relación residentes-turistas, concebidos cada uno de ellos como actores sociales, que participan en la interacción esperando un intercambio en el marco de la reciprocidad y en el que sólo se realiza y se mantiene la interacción turística cuando ambos actores perciben tras evaluar la relación, que obtendrán una recompensa esto es, que el balance será favorable (Ap, 1992).

La relación de intercambio en sí misma se compone de dos elementos: antecedentes y forma de la relación. En cuanto a los antecedentes, representan las oportunidades o situaciones en una relación de intercambio, percibidas como tales al menos por uno de los actores sociales. La búsqueda racional de una recompensa se explica en las comunidades receptoras de turismo en el sentido de que en la medida en la que se perciban mayores beneficios, más positiva será la percepción de involucrarse en la actividad turística, o en términos negativos, mientras mayores sean los costos percibidos, también será más negativa la percepción de la actividad turística. El antecedente del intercambio turístico se presenta por el lado de los residentes en una comunidad en la expectativa de que al atraer y desarrollar turismo mejoraría su bienestar económico, social y psicológico. Por el lado del turista, existen diversas motivaciones para visitar y realizar actividades de ocio en un determinado destino (Mathieson y Wall, 1982). El intercambio comienza cuando alguna de las partes busca satisfacer la necesidad o deseo acercándose a la contraparte. Los antecedentes se pueden comprender en una valoración conjunta de tres condiciones: la existencia de beneficios mutuos, la reciprocidad en el intercambio y el principio de justicia en el intercambio de beneficios.

La búsqueda de beneficios puede actuar a favor de la actividad turística aún cuando los beneficios máximos no se alcancen o incluso aunque existan altos costos, siempre que la percepción de los beneficios siga siendo mayor que ellos. La reciprocidad, es condición para el intercambio, consiste en que cada uno de los actores involucrados ofrece una retribución en términos de valor a su contraparte, en el caso del intercambio turista-residente, los residentes para estar en condiciones de aceptar la actividad turística tienen que percibir que ésta les aportará beneficios aproximadamente de la misma medida o del mismo valor que ellos aportarán, tanto en términos de recursos materiales como instalaciones y atractivos, como en términos intangibles como la hospitalidad, la cortesía, atención, servicio, entre otros. A su vez el turista también considerará en reciprocidad su entrada al intercambio en tanto perciba que los beneficios o recompensas que recibirá son equilibrados en relación con los que él mismo dispone para 
el intercambio, además del recurso económico, estarían incluidos el tiempo, el trato amable y cortés, el respeto a la cultura local, entre otros.

El principio de justicia es la tercera condición de los antecedentes a considerar en un intercambio balanceado, además de los beneficios y la reciprocidad, los actores involucrados evalúan si es que los beneficios a recibir son los adecuados, considerados como tales en su propio entorno. Toda vez que se cumplen los antecedentes en conjunto de manera favorable, la relación de intercambio toma forma, en tanto que si uno o más de los antecedentes se incumple, entonces la relación no toma forma.

$\mathrm{El}$ segundo elemento de la relación de intercambio lo constituye la forma de la relación, en función del poder que sobre la relación misma tenga cada uno de los actores, se puede formar de manera balanceada o desbalanceada a favor de uno u otro de los actores. Cuando se trata de una relación balanceada de intercambio, pueden darse dos formas, ya sea que ambos actores tienen elevado poder sobre la relación, lo que favorece la formación y mantenimiento de la relación, en tanto que cuando se trata de una relación en la que ambos actores tienen bajo poder sobre ella, resulta poco probable que se forme la relación pues hay bajo incentivo para ello, aún cuando fuese balanceado.

En el caso de las relaciones de intercambio desbalanceadas, también existen dos formas básicas, la desbalanceada a favor del turista y la desbalanceada a favor de los residentes, si bien la literatura en turismo desde Krippendorf (1987) ya se señalaba que en la mayor parte de los casos es el turista, por ser considerado quien paga y por tanto quien "siempre tiene la razón", también existe la posibilidad de que en algunos casos, momentos y contextos particulares sean los residentes quienes tengan un poder mayor sobre la relación de intercambio.

Para el caso que interesa en el presente documento, las relaciones de intercambio que se analizarán, son específicamente aquellas que se presentan de manera intencional, basadas ya sea en el intercambio comercial o basadas en el intercambio personal (Sharpley 2010). Lo anterior, con el objetivo de demostrar que el tripulante de crucero, a partir de una relación de intercambio constante con los residentes locales de Cozumel, generan efectos diferenciables de aquellos producidos por la interacción entre turistas y habitantes de la isla.

\section{Metodología}

Autores como Ap (1992); Brunt y Courtney (1999); Shanahan, Pelstring y McCommas (1999); Jennings (2001); Lepp (2006) y Vacilachis, 2006, argumentan que los métodos cualitativos permiten obtener información más detallada de los informantes sobre un tema en específico y con ello, comprender de mejor manera una realidad desde su perspectiva que utilizando una metodología cuantitativa. Por lo anterior, el trabajo de campo de la presente investigación (el cual se llevó a cabo de febrero de 2014 a marzo de 2016), constó de dos diferentes etapas cualitativas. La primera de ellas, consistió en la realización de entrevistas semiestructuradas dirigidas a tripulantes de cruceros. El guion se conformó de preguntas que permitieron identificar las actividades que el tripulante realiza durante su tiempo libre en la isla, así como averiguar qué lugares visitan y si lo hacen con frecuencia. Durante esta etapa de investigación, se logró entrevistar a 20 tripulantes (16 hombres y 4 mujeres). Debido a la pluriculturalidad de los trabajadores de cruceros, sólo 3 entrevistas fueron realizadas en español y el resto en idioma inglés, pues este último resultó ser un idioma que el tripulante domina y que permitió entablar en muchos casos una conversación fluida. Las entrevistas fueron realizadas en el mercado municipal, así como en distintos restaurantes, cafés, plazas y centros comerciales de la isla, principalmente en la zona centro y en los puntos de atraque de los cruceros.

Durante la segunda etapa, se entrevistaron a 17 residentes locales siendo 9 de los informantes de género masculino y 8 del femenino. Para lo anterior, fue necesario realizar un guion semiestructurado cuyo fin fue el conocer qué tan frecuente es su contacto con los tripulantes de cruceros y qué tipo de relación guardan con ellos. En esta fase de campo, se debe mencionar que 16 entrevistas fueron realizadas en español y una en inglés, ya que una residente no era nativa del país. Las entrevistas se realizaron en 16 diferentes restaurantes y cafés de la zona centro y sur de la isla, donde los entrevistados fungen como dueños o encargados de los negocios.

Todas las entrevistas realizadas durante las etapas de campo, fueron transcritas para posteriormente proceder al análisis de su contenido (Bardin, 2002) y así, poder comprender de mejor forma la interacción que existe entre los residentes locales de Cozumel con los tripulantes. 


\section{Resultados}

\subsection{Antecedentes de la interacción}

Durante el proceso de trabajo de campo, diversos informantes locales comentaron que el proceso de interacción comenzó principalmente cuando los tripulantes se acercaron a sus negocios para conseguir alimentos hace más de dos décadas. Sin embargo, ellos al provenir de distintas partes del mundo, buscaban platillos de su país de origen. A pesar de que por las diferencias de idioma (en algunos casos) la comunicación resultaba difícil, en muchos negocios de comida de la isla se llegó al acuerdo de que los dueños permitirían a los tripulantes hacer uso de los utensilios de cocina para que pudieran preparar sus alimentos.

"Mucha gente venía o quería comer lo que a ellos les gusta, se metían a la cocina y veíamos qué les ponían, se ponían el mandil y el gorro, y les dabas su tabla y pues nosotros "¿en qué te ayudamos?, ¿̇te limpiamos el pescado?" y bueno, es hasta el final que le ponen el ingrediente que va a dominar en los sabores", (Genaro, dueño, Restaurante El abuelo).

"Siempre pasaban los muchachos y vienen a buscar restaurantes y todo, pasaron con ella y se puso a atenderlos, los muchachos le hicieron plática y le dijeron que eran peruanos, y le preguntaron que si podían pasar a su cocina, y les dijo "Ahí está la cocina, pasen, ¿qué ingredientes necesitan?", no que pues lo típico, lo que es cebolla, chile, papa, carne, y pues la señora fue consiguiendo sus ingredientes y ella misma fue aprendiendo poco a poco". (Ana, encargada, Restaurante El Calamar).

"Fijate que ellos siempre fueron mis maestros [...] cuando yo inicié a trabajar en esto, en esa época hace 22 años se permitía entrar a las cocinas, o sea, se entraba a observar y ver como preparaban sus alimentos y ahi es donde yo aprendí a elaborarlos porque yo honestamente no sabía cómo elaborarlos, no sabía nada de ese tipo de comida [...] Entre medio inglés, medio español, y de su idioma de ellos, vas aprendiendo hasta cierto punto palabras de su dialecto, de su lengua, lo que te están pidiendo y vas aprendiendo de eso" (Mariana, dueña, Lonchería El Oasis).

Con el paso del tiempo, los dueños comenzaron a aprender a cocinar los alimentos y se comenzaron a preocupar por conseguir los ingredientes necesarios en supermercados locales, $u$ otros lugares como en la zona libre de Belice. En caso de no conseguirlos, el tripulante los traía de su país de origen o se sustituían por ingredientes similares.

"Son platillos que nos dicen que les preparemos, que con el paso de los años hemos aprendido a prepararlos igual, el lomo pues sí tenemos todos los ingredientes, que es la papa normal, la blanca, la amarilla, que son las mismas que tenemos en el mercado comúnmente, la cebolla, el tomate, la salsa soya, y pues todo se frie, todo lo que podemos encontrar en el súper ino?, con el tiempo fue mejorando y ellos mismos luego le ponen perejil, pero a veces no había perejil en el súper y lo cambiamos por cilantro". (Ana, encargada, Restaurante El Calamar).

"Ya hay una tienda especializada en condimentos o sea, que le vende productos a hoteles o a restaurantes, desde que abrió esa tienda pues ya nos facilitó mucho nuestro trabajo, antes muchos de los condimentos antes los traíamos anteriormente de la zona libre, o el mismo cliente a veces cuando ellos se van de vacaciones y regresan a trabajar ya traen condimentos de su lugar de origen”. (Gilberto, dueño, Lonchería Santa María).

"Tratamos de conseguir siempre la mayoría de ellos con tal de que sepa lo más similar a lo que ellos podrían comer en casa, compramos algunas cosas en el mercado, en el Chedraui... luego uno consigue buenas cosas ahi, en el Sam's club... hay algunos que no podemos de plano conseguir, pero utilizamos otros". (Norberto, gerente general, Fu-Lin Asian Cuisine).

Actualmente, en Cozumel se pueden encontrar restaurantes especializados en alimentos de distintas partes del mundo, principalmente, en frente de Puerta Maya y en el Mercado Municipal en el centro de la isla, donde se ofrecen platillos típicos de países como Filipinas, Indonesia, India, Jamaica y Perú (Ver fotografías 1,2 y 3 ). 
Fotografía 1: Tripulantes comiendo en el mercado municipal.

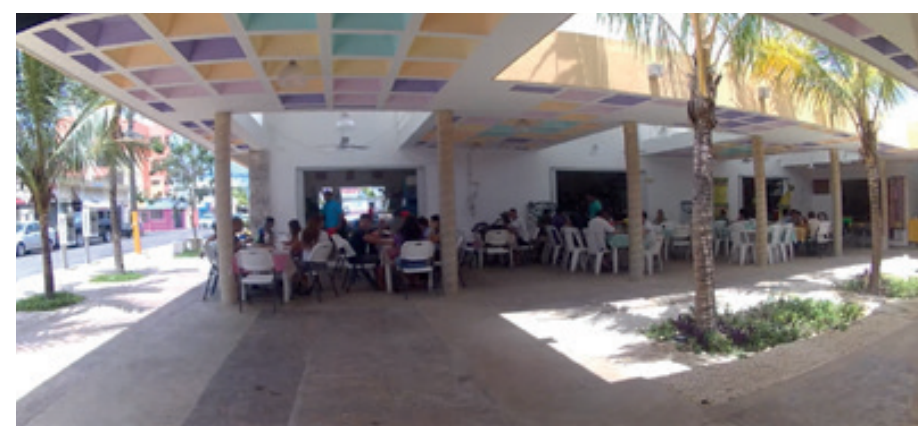

Fuente: Fotografía propia.

Fotografía 2: Restaurante de comida asiática Fu-Lin.

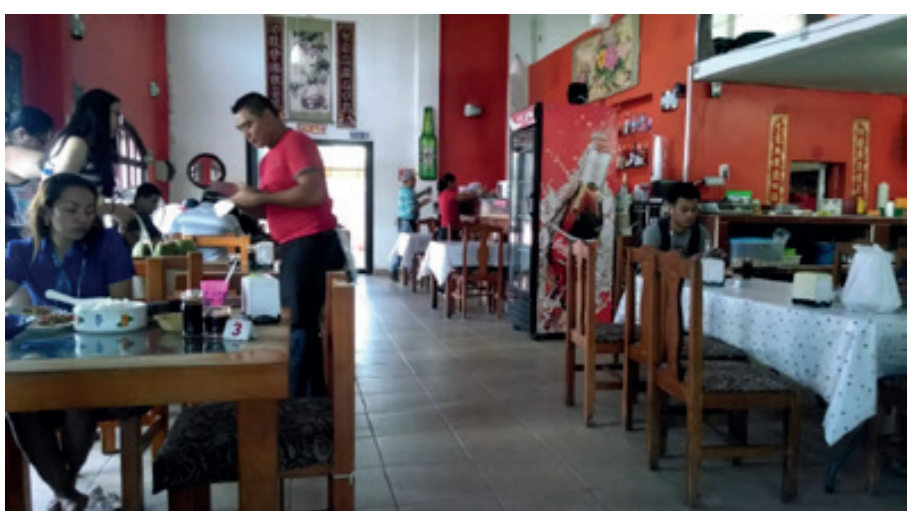

Fuente: Fotografía propia.

Fotografía 3: Restaurante de comida peruana y mexicana El Calamar.

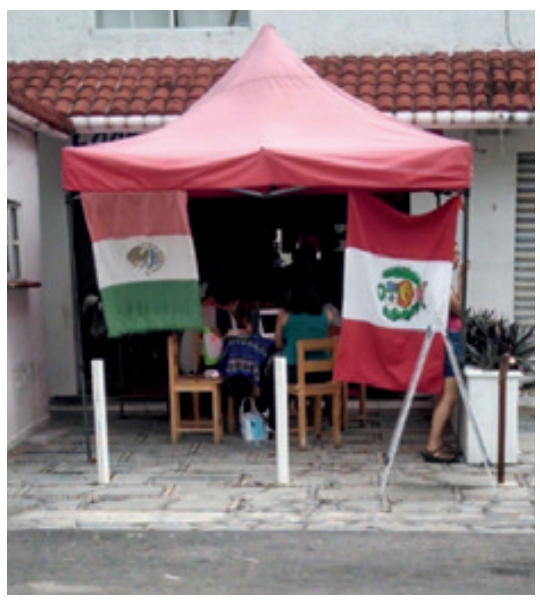

Fuente: Fotografía propia. 
Comentarios de algunos tripulantes permitieron confirmar que actualmente en el puerto les es muy sencillo encontrar alimentos de donde ellos provienen, principalmente para los tripulantes asiáticos, tal como Wong y Tuan mencionan.

"Yo usualmente voy a lugares de comida asiática como el restaurant Chi, ellos siempre tienen comida de Indoneisa y muchos tripulantes vamos ahí, es un sitio al que vamos frecuentemente". (Wong, aseo y limpieza, Filipinas).

"Algunas veces voy a comer a los restaurantes que se encuentran en frente porque nos gusta comer alimentos de donde somos, también porque tienen wi-fi gratuito y puedo hablar ahi con mi familia" (Tuan, coordinador de maniobras, Indonesia).

\subsection{Presencia del tripulante en la isla}

Si bien la mayoría de las interacciones entre tripulantes y residentes locales ocurren en sitios de venta de comida, también es una realidad que el tripulante tiene presencia en diversos lugares de Cozumel en los que hace uso de servicios dirigidos para la gente residente de la isla. Esto se hace evidente a partir de la constante visita a hospitales, consultorios dentales, estéticas de belleza y cafés internet. Evidencia de ello, son los comentarios realizados por David, Romina y Yessica.

"Yo fui la primer persona en el Cozumel Medical Center que sugirió introducir un convenio de servicio dental con las navieras, entonces yo sé todo el movimiento relacionado con los crews y el servicio de salud dental [...] estuve trabajando ahí por cinco años y pues la verdad después ocurrieron cosas ahí que ya no me gustaron mucho, tuvimos nuestras diferencias y me sali de ahi [...] ellos saben dónde y quién les ofrece un servicio de calidad, por eso es que aunque yo les cobre, prefieren venir conmigo". (David, dueño, Advanced dental care).

"Sí, mucho tiempo, como cuatro años y medio estuve trabajando con ellos (refiriéndose a los tripulantes). [...] Me cambié de lugar por lo mismo de que el lugar en el que estaba antes era muy pequeño, entonces en la ubicación en la que ahora estoy es un poquito más lejos, donde ellos ya no pasan [...] sí a algunos todavía los conservo como clientes que vienen cada tres meses o cuando les cambian su ruta es cuando dejan de venir". (Romina, dueña, Estética).

"Lo mínimo una hora, hay muchos que se quedan dos o tres horas (refiriéndose a los tripulantes), cuatro horas dependiendo del tiempo que tengan porque también tienen que llegar a tiempo al crucero a trabajar, entonces como les dan horarios, se vienen aqui al internet, se quedan un rato platicando con su familia, se van a comer, a comprar sus cositas y ya se regresan al barco". (Yessica, encargada, Café Internet Mexatel).

Incluso, un tripulante comentó que prefiere desplazarse hacia sitios donde normalmente transitan residentes locales, ya que afirma que en esos sitios se puede apreciar la vida típica de México.

"Al menos en mi caso, yo siempre busco más los lugares locales, algo más típico, lugares más auténticos, donde tu sabes, está el México real"(Christian, técnico de sistemas, Jamaica).

Así mismo, el tripulante durante su tiempo libre en el destino, puede llegar a hacer uso de servicios y lugares destinados a turistas, tales como arrendadoras de vehículos, clubes de playa, e incluso realizar excursiones. Los comentarios de Mario y Lucy a continuación, demuestran lo mencionado.

“Uff!, bastantes, obviamente acá hay otras compañias, pero viene mucho crew y viene por grupos, mira, por ejemplo, estos que vienen acá a simple vista son crews (refiriéndose a tres hombres y una mujer que entraron al local)". (Mario, encargado, arrendadora de autos Sol y Mar).

"Sí, van a la playa, está playa mía o San Francisco, se arman los tour, por ejemplo se van en autobús completo, inclusive Playa Mía o San Francisco... no sé si tienen quién les venda allá, pero les arman los paquetes, $y$ salen y ya, directo al autobús y se van, hay otros que arman directamente a Playa del Carmen o Xcaret" (Lucy, encargada, Estética Unisex Alexandra). 
$\mathrm{Al}$ preguntarle al tripulante Phill sobre si es verdad que realizan actividades dirigidas a los turistas, comentó que es común. Sin embargo, también aseguró que en muchos casos, es una actividad que no se hace con gran frecuencia, ya que deben volver a trabajar cuando suben al barco y buscan ahorrar el dinero que reciben de su trabajo.

"Las hacemos, ipor supuesto!, pero no siempre, quiero decir, no es una actividad frecuente... pero puedo apostarte que cualquier tripulante, sin importar su puesto en el barco o ingreso, lo ha hecho por lo menos una vez cuando vienen a Cozumel [...] no es siempre porque a veces simplemente preferimos relajarnos porque tenemos que volver al trabajo o porque queremos ahorrar un poco de dinero" (Phill, fotógrafo, Croacia).

Incluso existen sitios en la isla que fueron concebidos para principalmente atender al tripulante de crucero. El más destacado de ellos, es la plaza i95, propiedad de la empresa Wok on Roll, localizada en frente de Puerta Maya, donde existe un spa, un restaurante, un café internet, un mini super y un spa cuyo objetivo es satisfacer las necesidades del crew member durante su tiempo libre en la isla. Al respecto, Luisa comentó lo siguiente.

"Hace cerca de 4 años abrimos esta plaza, es nueva entre comillas, cuatro años, y también está enfocada totalmente al Crew Member, desde el Mini Súper, que no se si lo viste, el que está en la entrada, hay un mini súper, hay un café internet, tenemos dos restaurantes, uno que estamos a punto de abrir allá arriba, y el spa, que también es dirigido para tripulantes". (Luisa, gerente general, Wok on Roll).

\subsection{Opinión de los residentes locales sobre el tripulante}

Los residentes locales entrevistados consideran que el tripulante, de manera general, es un individuo trabajador que se ha visto en la necesidad de salir de su país de origen para proveer sustento a sus seres queridos. Esto ha generado empatía con los residentes locales que al igual que los tripulantes, trabajan duro con el objetivo de obtener ingresos para poder mantener a sus familias.

"Son personas muy buenas, trabajan mucho y como tú o como yo, buscan ganar buenos ingresos en su trabajo para poder darle una mejor vida a sus familiares... mucho de lo que ganan la verdad es que no se lo gastan... sino que lo mandan a sus casas". (Norberto, gerente general, Fu-Lin Asian Cuisine).

Es de conocimiento por parte de los residentes locales que el tripulante recibe un sueldo en los barcos. Lo anterior, ha generado una situación de abuso por parte de algunos sectores de la población, principalmente del servicio de taxis local, ya que varios entrevistados aseguran que las tarifas que manejan a los tripulantes son muy elevadas y que debería existir algún tipo de apoyo en su condición de trabajadores, sobre todo al tomar en cuenta que es un cliente que se tiene en la isla constantemente y que deja una derrama económica considerable.

"Ha habido muchas situaciones con los taxistas, su tarifa es de 8 dólares, les cobran 10, que si son más de 8 les cobran más de 16 dólares para ir al muelle internacional, o sea, hay muchas cosas que les hacen y pues

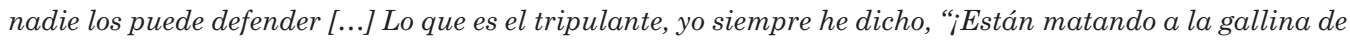
los huevos de oro!" porque el tripulante es fijo, lo tienen cada semana consumiendo por ocho meses, no hay ningún transporte público que los dejara en esta zona pon tú, a 2 dólares llegar al centro, no la hay, si viene una sola persona tiene que pagar 8 dólares del muelle al súper, 8 de regreso, son 16 dólares más lo que vaya a comprar en el súper y lo que vaya a gastar en comida y todo eso, cada semana, no les es redituable estar bajando cada semana”. (Mariana, dueña, Lonchería Oasis).

"Deberíamos optar por brindarles a ellos un tipo de apoyo en el sentido de un precio diferente al precio que le damos al turista, una persona que netamente viene a vacacionar, o sea, no darles las mismas tarifas a ellos como por ejemplo el servicio del taxi, ellos al final de cuentas son trabajadores, vienen a distraerse un poco acá pero también guardan para darlo a sus casas, o sea, no vienen con la mentalidad de gastarse todo lo que traen en el bolsillo, entonces si nos pusiéramos en ese plan yo creo que en ese sentido un mayor número de ellos optaría por salir cada vez que pegan en este puerto". (Gilberto, dueño, Lonchería Santa María). 
Esta situación fue confirmada por los mismos tripulantes, quienes aseguran que las tarifas impuestas los taxistas son un abuso para ellos, y que en ese sentido, la gestión local debería hacer algo al respecto.

"Las tarifas de los taxis son muy caras para el salario de un tripulante, porque, tu sabes, somos diferentes y quieren obtener dinero de nosotros, sin embargo, nosotros también venimos aquí por trabajo, por lo que sería genial tener otro tipo de enfoque”. (Mary, trabajadora de spa, Croacia).

Desde la perspectiva de algunos informantes que tienen contacto constante con el tripulante, consideran que el hecho de que tengan que ahorrar dinero para llevarlo a sus casas, ha generado en otros residentes la percepción de que el tripulante no gasta en el destino. Sin embargo, los mismos afirman que el tripulante es un individuo que deja muchos beneficios para el puerto de Cozumel al realizar distintas actividades en las que dejan una notable derrama económica, siendo en algunos casos, la fuente principal de sus ingresos.

\footnotetext{
"Son gente noble y trabajadora, no están fuera de sus casas por gusto, lo que si que muchas ocasiones se les discrimina porque pensamos que son muy baratos, que son <<piojos >> que no quieren pagar [...]en mi caso, ellos son la fuente de mi ingreso, si ellos no vinieran yo tendría que ver a qué otra cosa dedicarme, ellos no se quedan aquí a vivir, o sea, no le quitan las fuentes de empleo a nadie, al contrario, vienen a comentar que más negocios se abran”. (Gilberto, dueño, Lonchería Santa María).
}

"Muchos tripulantes bajan del barco en sus ratos libres y van y consumen diferentes cosas en la isla, ya sea comida, ropa, un café, lo que sea, pero el beneficio es notable ya que dejan una derrama económica en la isla”. (Jorge, encargado, Restaurante Rock n'Java).

"Pues el que viene a comer aquí luego compra fruta en el mercado, o que compra un par de zapatos aqui en frente, o que van a comprar perfumes con aromas de esos que preparan aquí por el Elektra, o que compran alguna vitamina, hay mucho beneficio". (Mariana, dueña, Lonchería Oasis).

"Muchos no vienen sólo aquí, que van al mercado, a la mega, al Chedraui... muchos, sobre todo los que ganan más, van a la playa o rentan un jeep y le dan la vuelta a la isla y hacen todo lo que quieran, pero pues entonces están dejando aquí y allá, y pues como es un cliente de cada 8 días, pues por supuesto que representa un beneficio para muchos de los negocios de la isla". (Norberto, gerente general, Fu-Lin Asian Cuisine).

Incluso un tripulante afirmó que cualquiera de ellos que descienda del barco en Cozumel, es seguro que dejará alguna derrama económica y que pueden llegar a gastar más dinero que los cruceristas.

"Te voy a decir algo, cada que un tripulante desciende del barco jes seguro que va a gastar en algo! Va a comer, comprar ropa, a tomar con los amigos, no lo sé... pero te aseguro que muchas veces los tripulantes gastan más dinero que los turistas (refiriéndose a los cruceristas) iPregúntales! Te aseguro que te dirán que gastan más de 50 dólares en cada ocasión (refiriéndose a los tripulantes). (Christian, técnico de sistemas, Jamaica).

\subsection{Mas allá de meras relaciones comerciales}

Las características de la visita del tripulante han propiciado que la interacción entre residentes y tripulantes no sean meramente transacciones económicas, ya que algunos informantes locales aseguran que han entablado relaciones de amistad con tripulantes que visitan constantemente sus negocios y que en caso de que el tripulante emprenda una ruta que no contemple al puerto de Cozumel por mucho tiempo, ellos vuelven sólo para visitarlos.

"A veces vienen y te empiezan a contar de sus familias, quieren a alguien con quien platicar, hasta me platican "este es mi hijo", "esta es mi esposa", es un complemento no solamente es la venta de los alimentos, porque te vuelves una amistad para ellos.[...] Pues el trabajar con el tripulante, como son clientela repetitiva porque los tienes cada semana o cada dos semanas, pues llegas a hacer cierta amistad con ellos, entonces muchas veces te traen recuerdos, otras veces se toman fotos contigo y ya que tuvieron tiempo vinieron y la traen impresa en un marco y te la obsequian, te invitan de comer, pues llegas a tener cierto lazo con ellos ¿no?”. (Gilberto, Dueño, Lonchería Santa María). 
"Hay gente que se va por mucho tiempo a otros lados, se pueden ir al Mediterráneo, se pueden ir a la parte del Caribe oriental, desaparecen cuatro, cinco años... y cuando regresan vienen otra vez a vernos, son los mismos empleados, la misma gente, y es bonito ino?, siempre se acuerdan de nosotros”. (Genaro, dueño, Restaurante El Abuelo).

"Yo tengo a varios amigos tripulantes peruanos, por ejemplo en el face, son muy platicadores, de hecho luego están en su país natal, en Perú y me escriben “'hola amiga como estás!, ¿Qué tal va todo en el Calamar?”... hace poquito pasó mi cumpleaños y se acordaron y me felicitaron, o sea, son amistades que aunque no nos veamos seguido ahi estamos en contacto en el internet. (Ana, encargada, Restaurante El Calamar).

Entablar relaciones de amistad ha beneficiado al tripulante en el sentido de que dueños de varios negocios, les realizan favores que van desde reservar un lugar en su restaurante, realizarles platillos especiales, hacerles descuentos especiales, entregar paquetes a tripulantes de otros barcos, hasta hacerles incluso la despensa.

"Pues tengo muchos clientes que por ejemplo nos dicen "Vamos a ir tal fecha" y hacen reservaciones, o hay muchas veces que llegan y te dicen "Oigan, necesito un favor, necesito recoger tal cosa de tal paquetería, ¿pueden ir por ella? O sea, ese tipo de cosas, o también te dicen que si le puedes dar una cosa a un amigo que viene en otro barco, entonces hay esa como confianza entre los tripulantes y los que trabajamos aquí, o sea, no solamente yo, sino todos los que trabajamos aquí". (Luisa, gerente, Wok on Roll).

"Nosotros les podemos hacer sus compras en el súper para que ellos puedan aprovechar su día, me la mandan por mail un día antes, yo voy al súper, me encargan sus cosas y se las tengo aquí listas para que se las puedan llevar, pueden hacer intercambio de cosas entre ellos, entre barcos... muchas veces uno deja una cosa de un barco y tiene un amigo y le quiere dar algo, no sé, y pueden dejar un paquete para la persona que venga en el barco de mañana o así, y también tenemos un servicio en el que si ellos me mandan su receta y la lista o la cantidad de gentes que van a venir a comer nosotros les preparamos su comida, por ejemplo, si fueran de la india o filipinos o algo así”. (Karina, dueña, Restaurante 10 con 15).

\subsection{Tripulantes residiendo en Cozumel}

Las entrevistas permitieron identificar que Cozumel es un puerto muy del agrado del tripulante, de tal forma que muchos de ellos han preguntado qué tan costoso resulta vivir en la isla, tal como comentó Yessica.

"Les gusta mucho, muchos hasta me preguntan que qué tal está en cuestión de comprar casa y todo eso, a veces les comento que para vivir Cozumel es caro [...] luego algunos quieren saber cuánto cuesta una casa o rentarla, hay algunos que piensan venir con su familia en vacaciones, algunos ya quieren comprar una casa, algunos quieren ya venirse para acá, les gusta mucho la isla”. (Yessica, Encargada, Mexatel).

Así mismo, se lograron identificar dos casos en los que los entrevistados solían ser tripulantes que actualmente radican en Cozumel y han formado una familia. Uno de ellos es Luis, quien dijo ser un tripulante jubilado que lleva 29 años radicando en la isla. Actualmente se encuentra casado y tiene un hijo con una residente local. Por otro lado, la informante Karina, tiene cuatro años en la isla, tiene un hijo y es la dueña del restaurant 10 con 15, mismo que constantemente recibe tripulantes como clientela.

"Estoy casado, ¿conoces a mi esposa?... hemos estado casados desde 1987, aún tengo mi acta de matrimonio [...] Bueno, esa fue la primera vez que estuve en Cozumel, eso fue en Julio de 1987 y era mi cumpleaños y dije (moviendo su cabeza y riéndose viendo a su esposa) isí [...] Y en ese momento empezamos nuestra relación, y yo llegaba aquí cada Lunes, y el barco en ese entonces se iba a la media noche, así que durante el día venía aquí (refiriéndose a su casa), y a las once en punto, tenía que regresar al barco". (Luis, Jefe de meseros, Filipinas).

"Hace diez años trabajé para Royal Caribbean, como youth staff cuidando niños dentro de las navieras por tres años y medio [...] Mi marido vive aquí desde hace ocho años y nos reencontramos vía Facebook hace cuatro, y poco tiempo después decidí casarme y venirme para acá a vivir con él [...] Cuando tuvimos la oportunidad de comprar el restaurante, pues es una oportunidad que se nos dio para también poder estar 
con nuestra hija, para poder pasar tiempo en familia, trabajamos juntos mi marido y yo, entonces eso nos da chance de marcar nuestro propio horario y... alargarlo a los crew members es una oportunidad más de obtener clientes". (Karina, Dueña, Restaurante 10 con 15).

Así mismo, Karina manifestó no ser un caso único de matrimonio entre tripulantes en Cozumel, ya que ella conoce a una pareja de tripulantes casados, que residen en la isla y que tuvieron un hijo con nacionalidad mexicana.

"De hecho tenemos una chica que trabajó en cruceros y que de hecho su marido sigue trabajando en el crucero, pero pues como viven aquí en Cozumel [...] él es el único que todavía sube y baja en barcos, él es de Ucrania y ella es de Sudáfrica [...] Ya viven aquí, acaban de tener un bebé ayer, imexicano claro!". (Karina, Dueña, Restaurante 10 con 15).

\section{Discusión}

\subsection{Interacción entre residentes y tripulantes con fines económicos}

Ante la expectativa de obtener beneficios económicos que le permitirán sostener sus negocios, la población residente otorga valor a la formación de relaciones de intercambio con los tripulantes de los cruceros. A su vez, los tripulantes de los cruceros valoran la posibilidad de obtener bienes y servicios a costos menores que los que tienen que pagar los pasajeros del crucero, por lo que también tienen la disposición a favor de entablar relaciones de intercambio con los residentes locales

Las repercusiones económicas que el turismo de cruceros puede ocasionar en los puertos de escala, han sido destacadas por Brida y Zapata, (2010), Kerswill y Mair (2015) y Polat (2015); pero cabe resaltar que dichos trabajos se centran en la interacción que existe entre locales y cruceristas, o viendo como similares los beneficios que pueden existir con los tripulantes de cruceros.

A diferencia de las actividades que normalmente realiza el crucerista (Brida y Zapata, 2010; Thyne et al., 2015), los resultados del presente trabajo permiten evidenciar que para muchos locales del puerto de escala de Cozumel, el tripulante de crucero es un visitante que representa un beneficio para distintos sectores, siendo evidente, una derrama económica que retribuye directamente en negocios que en su mayoría son gestionados por residentes locales. Por lo anterior, sería valioso realizar un trabajo en el que se especifique los efectos económicos generados por el tripulante puntualizando el número y giro de negocios a los que suelen acudir, para con ello, obtener un mejor conocimiento del número de residentes locales beneficiados por su visita.

\subsection{Interacciones Sociales}

Si bien ya se ha mencionado que el contacto entre trabajadores de cruceros y la población local ocurre principalmente en sitios donde se ofrecen alimentos y bebidas (Van Broeck y Dierckx, 2011), es necesario mencionar que es una realidad que no solo personas con antecedentes laborales en los cruceros, o del mismo origen étnico del tripulante, les ofrecen platillos típicos, sino que también los residentes locales se empeñaron en conocer y aprender recetas de comida del tripulante sin importar su país de origen; en primer instancia, al verlo como un cliente potencial y posteriormente, al sentir empatía por su condición de trabajadores en muchos casos.

$\mathrm{Al}$ igual que en el caso de los efectos económicos, la literatura ha catalogado los efectos sociales entre el local y tripulante como homólogos de aquellos derivados de la interacción con cruceristas (Gibson y Bentley, 2007; Polat, 2015). Sin embargo, es necesario destacar que a partir del contacto constante entre el tripulante y el residente local, se han formado en ocasiones lazos de amistad duraderos, lo cual difiere del contacto que normalmente existe entre turistas y locales, demostrando que pueden existir relaciones en los destinos turísticos que no necesariamente culminen en una mera transacción de compra-venta de bienes y servicios (Ribes y Pulido, 2015).

La existencia de relaciones de intercambio sostenidas a lo largo del tiempo, se sustenta en que los antecedentes de la relación cumplen con las tres condiciones previstas en la teoría: la existencia de beneficios mutuos, la reciprocidad en el intercambio y el principio de justicia en el intercambio de beneficios.

Tanto tripulantes como población residente tienen como antecedente la posible existencia de beneficios económicos mutuos, pero también esperan que la relación sea recíproca y que los beneficios sean intercambiados de manera justa, no sólo en términos económicos, puesto que si bien cada uno 
de estos dos grupos sociales tiene grupos de referencia culturales distintos, ambos se identifican como trabajadores asalariados o pequeños propietarios, que merecen y buscan un trato justo y la posibilidad de ahorrar, así como la posibilidad de reafirmar su posición social como trabajadores.

El hecho de que existen tripulantes que han optado por ver a Cozumel como un lugar para casarse, vivir y formar una familia (independientemente de su país de origen), evidencia un fenómeno de interculturalidad que no solo ocurre dentro del barco (Wood, 2000; Gibson, 2008), ni únicamente derivado de la relación entre turistas y locales (Polat, 2015), sino también derivado de la presencia de tripulantes en los puertos de escala que visitan, misma razón por la que se hace necesario profundizar aún más en cuestión de los efectos sociales del tripulante de crucero en los diversos puertos de escala donde tienen presencia.

\section{Conclusiones}

Con la evidencia presentada, puede concluirse que las relaciones sociales que se producen entre la población residente y los miembros de la tripulación de los cruceros muestran una naturaleza distinta a aquellos que se producen con los pasajeros de los cruceros y que tienen una tendencia a establecerse como relaciones duraderas al ser analizadas desde la teoría del intercambio social. Si bien se pueden enmarcar en el ámbito de los impactos sociales del turismo, la forma en la que operan es mucho más parecida a la de los puertos mercantes, ya que el tripulante tiene contacto constante con los residentes locales al desembarcar cada ocho días en el mismo puerto, al menos durante ocho meses.

A partir de la teoría de intercambio social, se puede confirmar que la interacción entre tripulantes y residentes locales nace primeramente por un intercambio comercial de bienes y servicios que, a diferencia de la interacción con los turistas de crucero, se ha vuelto constante debido a la visita regular del tripulante en el destino. Lo anterior ha generado que, al paso del tiempo, exista una condición de reciprocidad retroalimentada por la identificación social con el rol de trabajador tanto del tripulante como del residente local que lo atiende. En ese sentido, existen casos de relaciones sociales donde la reciprocidad se mantiene en balance, permitiendo la formación de amistades e incluso parejas formales entre ambos grupos sociales.

Derivado de lo anterior, se puede inferir que de la relación entre tripulantes y residentes locales pueden surgir diversos efectos sociales y económicos muy específicos que requieren mayor estudio, mostrando de esta manera la influencia que pueden tener los tripulantes de crucero en los puertos que visitan durante su tiempo libre. Si bien esto puede ser demostrado a partir de la teoría implementada, son necesarios más trabajos académicos con distintos enfoques y teorías, aplicados a diversos destinos de escala de cruceros turísticos, que evidencien la importancia que tiene la presencia del tripulante, la cual supera en posibilidades de establecerse a la que significa la presencia del pasajero o turista.

Mención adicional merecen las diferencias socioculturales que, si bien en principio pueden ser vistos como posibles barreras para el establecimiento de relaciones duraderas, como el idioma, las costumbres, la gastronomía, la religión y los valores, las creencias y tradiciones, en los casos observados, con el paso del tiempo tales diferencias se allanan, se diluyen y se conducen hacia puntos de encuentro, conformando posiblemente pequeñas comunidades interculturales. Ante estas situaciones, saltan las interrogantes respecto a ¿qué sucederá con estas comunidades en el futuro? ¿tenderán a crecer y a mantenerse integradas a su interior y en relación con la comunidad residente o existen posibilidades de conflicto y de sus efectos negativos como la marginación o la violencia por segregación? ¿Hasta dónde estas condiciones reflejan la cultura organizacional de las empresas navieras y/o sus políticas de integración de sus tripulaciones? ¿Hasta dónde llegaría la responsabilidad de atención y cuidado de estas comunidades por parte de las autoridades locales o de las propias empresas navieras? Estas preguntas por ahora permanecen sin respuesta, pero sin duda pueden tomarse como punto de partida para futuros estudios en la temática.

\section{Bibliografia}

Administración Portuaria Integral de Quintana Roo.

2015. APIQROO, Estadísticas. Disponible en: http://www.apiqroo.com.mx/estadistica/. Consultado el: 05/03/2015.

Andereck, K. L., Valentine, K. M., Knopf, R. C., \& Vogt, C.

2005. Residents' perceptions of community tourism impacts. Annals of Tourism Research, 32(4): 1056-1076. 
Ap, J.

1992. Residents' perceptions on tourism impacts. Annals of Tourism Research, 19(4): 665-690.

Axelrod, R.

1984. The evolution of cooperation. Nueva York, NY, E.U.: Basic Books.

Bardin, L.

2002. Análisis de contenido. Madrid, España: Ediciones Akal.

Blau, P.

1964. Exchange and power in social life. Nueva York, NY, E.U.: Wiley.

Boy, C. y Neumann, S.

2012. Regulatory Frameworks of the Cruise Industry. En: Vogel, M., Pathanassis, A. Y Wolber, B. The

Business and Management of Ocean Cruises. Wallingford, UK: CAB International.

Brida, J., y Zapata, S.

2010. Cruise Tourism: Economic, Socio-Cultural and Environmental Impacts. International Journal of

Leisure and Tourism Marketing, 1(3): 205-226.

Brunt, P., y Courtney, P.

1999. Host perceptions of sociocultural impacts. Annals of tourism Research, 26(3): 493-515.

Business Research \& Economic Advisors

2015. Economic Contribution of Cruise Tourism to the Destination Economies, Vol.I \& II. Exton, USA: BREA.

Crew Center

2017. Find out: How many crew members work in the cruise industry? Disponible en: http://crew-center. com/find-out-how-many-crew-members-work-cruise-industry. Consultado el: 04/03/2017.

Cruise Lines International Association Inc.

2017. 2017 CLIA State of the Industry. Disponible en: http://www.cruising.org/about-the-industry/ research/2017-state-of-the-industry. Consultado el: 10/01/2017.

Dowling, R.

2006. The Cruising Industry. En: Dowling, R. Cruise Ship Tourism. London, UK: CABI.

Dugatkin, L. A.

1977. Partner choice, game theory and social behavior. Journal of Quantitative Anthropology, 5: 3-14.

Edwards, W.

1954. The theory of decision making. Psychological Bulletin, 51, 380-417. http://dx.doi.org/10.1037/ h0053870

Florida-Caribbean Cruise Association

2015. Cruise Industry Overview, State of the Cruise Industry. Pembroke Pines, Florida, FCCA.

García, E. (2015). Comportamiento de los tripulantes de cruceros en Cozumel, México. Revista Turismo y Desarrollo Local TURyDES, 8(19): 1-8.

Gibson, P.

2008. Cruising in the 21st century, who works while others play? International Journal of Hospitality Management, 27(1): 42-52.

Gibson, P., y Bentley, M.

2006. A Study of Impacts: Cruise Tourism and the South West of England. Journal of Travel and Tourism Marketing, 20: 63-77.

Henry, J., \& Thyne, M.

2007. Getting them off the Boat: An Exploratory Study into Cruise Ship Passenger and Crew Motivations to

Disembark. In Australian and New Zealand Marketing Academy, ANZMAC Conference .2007: 684-691.

Homans, G. C.

1961. Social behavior: Its elementary forms. Nueva York, NY, E.U.: Harcourt Brace \& Jovanovich.

Instituto Nacional de Estadística y Geografía

2015. Panorama Socioeconómino del Estado de Quintana Roo. Disponible en: http://www.inegi.org. $\mathrm{mx} /$ prod_serv/contenidos/espanol/bvinegi/productos/ce nsos/poblacion/2010/panora_socio/qroo/

Panorama_QRoo.pdf. Consultado el: 2/07/2015

Jennings, G.

2001. Tourism Research. Milton: John Wiley \& Sons.

Johnson, D.

2002. Environmentally Sustainable Cruise Tourism: A Reality Check. Marine Policy, 26: 261-270. 
Kantor, J. R.

1959. Interbehavioral psychology. Chicago, IL, E.U.: Principia Press.

Kerswill, M., y Mair, H.

2015. Big Ships, Small Towns: Understanding Cruise Port Development in Falmouth, Jamaica. Tourism in Marine Environments, 10(3-4): 189-199.

Klein, R.

2003. Cruising-Out of Control: The Cruise Industry, The environment, Workers, and the Maritimes. Canadian Centre for Policy Alternatives. 27.

Klein, R.

2011. Responsible Cruise Tourism: Issues of Cruise Tourism and Sustainability. Journal of Hospitality and Tourism Management, 18: 107-116.

Krippendorf, J.

2010. The Holiday makers. Oxford: Butterworth-Heinemann.

Lepp, A.

2006. Residents' attitudes towards tourism in Bigodi Village, Uganda. Tourism Management, 28: 876-885.

Lloyd, N., Henry, J. y Thyne, M.

2011. Do Passengers Listen to their Crew? The Use of Word-of-Mouth Recommendations on Board a Cruise Ship. Journal of Hospitality and Tourism Management, 18: 88-94.

Lois, P., Wang, J., Wall, A. y Ruxton, T.

2004. Formal Safety Assessment of cruise ships. Tourism Management, 25: 93-109.

Martínez. C.

2008. Configuración Territorial del Turismo en las Costas de la Isla de Cozumel. Teoría y Práxis, 5: 343-357.

Mathieson, A., y G. Wall

1982. Tourism: Economic, Physical and Social Impacts. New York: Longman.

Monterrubio, J. C., Sosa, A. P., \& Josiam, B. M. (2014). Spring break e impacto social en Cancún, México. Un estudio para la gestión del turismo. Anuario Turismo Y Sociedad Universidad Externado de Colombia, 15: 149-166.

Morales Domínguez, J. F.

1978. La teoría del intercambio social desde la perspectiva de Blau. Revista Española de Investigaciones Sociológicas - REIS, 1(4): 129-146.

Palafox, A., Aguilar, A., y Anaya, J.

2015. Cozumel y la Transformación de su Paisaje por el Turismo de Cruceros. Revista de Ciencias Sociales, 149: 103-115.

Petrick, J., y Durko, A.

2015. Segmenting Luxury Cruise Tourists Based on their Motivations. Tourism in Marine Environments, 10, (3-4): 149-157.

Polat, N.

2015. Technical Innovations in Cruise Tourism and Results of Sustainability. Procedia, Social and Behavioral Sciences, 195: 438-445.

Ribes, E.

2001. Functional dimensions of social behavior: Theoretical considerations and some preliminary data. Revista Mexicana de Análisis de la Conducta, 27: 285-306.

Ribes, E., \& López, F.

1985. Teoría de la conducta: un análisis de campo y paramétrico. México, D.F.: Trillas.

Ribes, E., \& Pulido, L.

2015. Reciprocidad, Tipos De Contingencias Sociales Sistémicas Y Lenguaje: Investigación De Las Interacciones Interindividuales. Revista Mexicana de Psicología, 32(1): 81-91.

Santander, L. y Ramos, M.

2011. El nacimiento de un destino turístico en el Caribe Mexicano. Cozumel, de Isla abandonada a puerto de cruceros. El Periplo Sustentable, 21: 5-30.

Shanahan, J., Pelstring, L. y McComas, K.

1999. Using Narratives to think about environmental attitude and behavior: An exploratory study.

Society and Natural Resources, 12: 405-419.

Sharpley, R.

2014. Host perceptions of tourism: A review of the research. Tourism Management, 42: 37-49. 
Thibaut, J. \& Kelley, H.

1959. The social psychology of groups. Nueva York, NY, E.U.: Wiley.

Thyne, M., James, H., \& Lloyd, N.

2015. Land Ahoy, How Cruise Passengers Decide on Their Shore Experience. Tourism in Marine Environments, 10, (3-4): 177-187.

Vacilachis, I.

2006. Estrategias de Investigación Cualitativa. Argentina, Gedisa.

Van Broeck, A., y Dierckx, G.

2011. Activities of cruise ship employees in Cozumel, Mexico. Études Caribéennes, Disponible en: http: // etudescaribeennes.revues.org /5133?lang=es. Consultado el: 06/01/15.

Véronneau, S., y Roy, J.

2012. Cruise Lines' Purchasing and Logistics Management. En: Vogel, M., Pathanassis, A. Y Wolber, B. The Business and Management of Ocean Cruises. Wallingford, UK: CAB International.

Weaver, A.

2005. Interactive service work and performative methaphors: The case of the Cruise Industry. Tourist Studies, 5, (1): 5-27.

Wood, R.

2000. Caribbean Cruise Tourism, Gobalization at sea. Annals of Tourism Research, 27 (2): 345-370.

$\mathrm{Wu}, \mathrm{B}$.

2005. The world Cruise Industry: Aprofile of the Global Labour Market. Cardiff, UK, Seaferers International Research Centre.

Zhao, M.

2002. Emotional Labour in a Globalized Labour Market: Seaferers on Cruise Ships. Seaferers International Research Centre. Cardiff, UK. 